\title{
Predicted effects of parallel temperature gradients on the overestimation of TCV divertor target Langmuir probe $T_{e}$ measurements
}

\author{
J. Horacek ${ }^{*}$, R.A. Pitts, P. C. Stangeby ${ }^{\mathrm{a}}$, O. Batishchev ${ }^{\mathrm{b}}$, A. Loarte ${ }^{\mathrm{c}}$ \\ CRPP-EPFL Lausanne, CH-1015, Switzerland \\ ${ }^{a}$ University of Toronto, Institute for Aerospace Studies, Canada. \\ ${ }^{b}$ Lodestar/MIT, Cambridge, MA 02139, USA \\ ${ }^{c}$ EFDA-CSU, Max Planck Institut für Plasmaphysik, D-85748 Garching, Germany
}

\begin{abstract}
During high recycling conditions and divertor detachment in the TCV tokamak, divertor target Langmuir probes never measure electron temperatures much below $\sim 5 \mathrm{eV}$. Code simulations using $\mathrm{B} 2$-Eirene of these plasmas indicate that $\mathrm{T}_{\mathrm{e}} \approx 1 \mathrm{eV}$. This inconsistency has also been seen in other tokamaks. In this paper, the influence on a theoretical Langmuir probe VI-characteristic of a strong $\mathrm{T}_{\mathrm{e}}$ gradient parallel to the magnetic field is examined by using B2-Eirene simulated $\mathrm{T}_{\mathrm{e}}$ and $\mathrm{n}_{\mathrm{e}}$ profiles and adopting a simple numerical approach to compute divertor target electron energy distribution functions. Standard fitting of these theoretically generated VI-characteristics results in a temperature very close to the predicted value at the highest densities corresponding to divertor detachment in TCV. At intermediate densities, the computed temperature at the inner target is found to be very sensitive to assumptions regarding the magnitude of the assumed electron collision mean-free-path.
\end{abstract}

\section{Introduction}

It is frequently observed, but infrequently reported, that tokamak divertor target Langmuir probes under high recycling and detached conditions yield electron temperatures higher than those measured by alternative methods, e.g. spectroscopy, Thomson scattering and thermography, see e.g. [1]. In particular, the very low $\mathrm{T}_{\mathrm{e}}(\sim 1 \mathrm{eV})$ associated with strongly recombining detached or partially detached divertor plasmas are often not reproduced by probes, with values in the range $\mathrm{T}_{\mathrm{e}} \sim 5 \mathrm{eV}$ being typical. This is regularly experienced in the TCV tokamak [2]. In such plasmas, strong temperature and density gradients parallel to the magnetic field arise which may perturb the form of the target electron energy distribution function (EDF), particu-

*. Permanent address: http://go.to/horacek/ 
larly at high energies, since faster electrons originating further upstream of the divertor may travel collisionlessly to the targets. Because Langmuir probes are sensitive only to the high energy tail of the distribution, such distortions can lead to overestimated probe $\mathrm{T}_{\mathrm{e}}$ and subsequent underestimation of the local density ( $\operatorname{since} \mathrm{n}_{\mathrm{e}} \propto \mathrm{T}_{\mathrm{e}}{ }^{-1 / 2}$ ). A number of authors have addressed this question theoretically [3-9].

Recently [3], a simplified kinetic approach adopted in [5] describing such $\mathrm{T}_{\mathrm{e}}$ overestimation, has been modified by introducing an adjustment to the electron mean-free-path, $\lambda \propto T^{2} / n$, assumed in [5] and by introducing a simple technique for numerical construction of the target EDF which can then be used to derive the expected Langmuir probe characteristic. The model requires the parallel $T_{e}$ and $n_{e}$ profiles as input. These are currently unavailable on TCV and so this paper uses B2-Eirene simulations of the parallel profiles in conjunction with the simple kinetic model to examine theoretically the impact of fast electrons on TCV target probe $\mathrm{T}_{\mathrm{e}}$.

\section{Experimental characteristics and B2-Eirene code modelling}

The TCV tokamak $\left(R=0.89 \mathrm{~m}, \mathrm{a}=0.25 \mathrm{~m}, \mathrm{~B}_{\varphi}=1.43 \mathrm{~T}\right)$ is a medium-sized device with an open divertor and highly unconventional, variable divertor geometries [2]. In the equilibrium of Fig.1, one of the standard TCV single-null lower diverted configurations, the poloidally long outer divertor leg reaches conditions of high recycling at medium upstream plasma densities, with partial detachment occurring first at the strike point as the density is increased further. For the standard operating condition on $\mathrm{TCV}$, with the $\mathrm{Bx} \nabla \mathrm{B}$ drift direction away from the $\mathrm{X}$ point, the inner divertor tends to be hotter and begins to detach only at the highest densities.

In Fig.2a, several experimental divertor LP VI-characteristics are compiled from probes near the inner and outer strike points during a TCV ohmic density ramp discharge with magnetic equilibrium shown in Fig.1. The strong outer target strike point detachment is clearly visible at high plasma density. At the inner target, detachment at the strike point occurs only at the highest densities. At the outer target strike point, $\mathrm{T}_{\mathrm{e}}$ never drops below $\sim 5 \mathrm{eV}$ and never below $\sim 10 \mathrm{eV}$ at the inner strike point. One should also note in addition that at high density, the ratio of ion to electron saturation currents at the outer target probe approaches unity and the characteristics diverge in many cases strongly from exponential behaviour.

The B2-Eirene code [10] is routinely applied to modelling of detachment in TCV [2]. Parallel field $\mathrm{T}_{\mathrm{e}}$ and $\mathrm{n}_{\mathrm{e}}$ profiles at various radii in the scrape-off layer (SOL) are an automatic result of any converged solution. In this paper, results from the SOLPS4 B2-Eirene package are used 
with no drifts included and with carbon as the only impurity species. Fig.3 compiles profiles, $\mathrm{T}_{\mathrm{e}}(\mathrm{x})$ and $\mathrm{n}_{\mathrm{e}}(\mathrm{x})$, with $\mathrm{x}$ the coordinate along the magnetic field, corresponding to the flux surface at distance of $1.8 \mathrm{~mm}$ outside the outer midplane separatrix (see Fig.1). These simulated $\mathrm{T}_{\mathrm{e}}(\mathrm{x})$ and $\mathrm{n}_{\mathrm{e}}(\mathrm{x})$ profiles are used as input to the kinetic analysis described below in Section 3.

At the outer divertor the high recycling state is achieved in the simulation already at low upstream density. Detachment follows as density is increased further. One may note in the higher density cases an extended "convective" region below the $\mathrm{X}$-point in which $\mathrm{T}_{\mathrm{e}}(\mathrm{x})$ is flat, and the low predicted value of $\mathrm{T}_{\mathrm{e}}$ at both inner and outer targets in the range $0.6->1.5 \mathrm{eV}$. This convective zone has been directly measured using divertor Thomson scattering on the DIII-D tokamak [11].

\section{Outline of numerical approach to modelling the target probe characteristic}

Stangeby has recently proposed [3] some modifications to the analytic approach due to Wesson [5] providing a description of the target EDF in the presence of a parallel $\mathrm{T}_{\mathrm{e}}$ gradient. A numerical approach is adopted in which electrons are launched from the target back upstream with given kinetic energy, $\varepsilon_{0}$ (in fact, in this paper "target" is taken to imply at the sheath edge). Their collision probability is computed for small steps along the field (accounting for the variations in $\mathrm{T}_{\mathrm{e}}, \mathrm{n}_{\mathrm{e}}$ and plasma potential, $\phi$ ) until the sum of these probabilities reaches unity. This defines a distance, $\mathrm{x}_{\mathrm{c}}$, at the last-collision as $\mathrm{p}\left(\mathrm{x}_{\mathrm{c}}, \varepsilon_{0}\right)=1$ where the collision probability is

$$
\mathrm{p}\left(\mathrm{x}_{\mathrm{c}}, \varepsilon_{0}\right)=\int_{0}^{\mathrm{x}_{\mathrm{c}}} \frac{\mathrm{dx}}{\lambda\left(\varepsilon_{0}+\phi(\mathrm{x}), \mathrm{T}(\mathrm{x}), \mathrm{n}(\mathrm{x})\right)} .
$$

More detailed discussion on the choice of $\lambda=\lambda(\varepsilon, T, n)$ can be found in [3]. Variation in $\phi$ is allowed for using energy conservation: $\varepsilon(x)=\varepsilon_{0}+e \phi(x)$. In common with Wesson [5], a Maxwellian $\mathrm{EDF}, \mathrm{f}^{\mathrm{M}}$, is assumed to exist at every point on the parallel profile. The target EDF can be then evaluated as $\mathrm{f}\left(\varepsilon_{0}\right)=\mathrm{f}^{\mathrm{M}}(\varepsilon(\mathrm{x}))$ :

$$
f\left(\varepsilon_{0}\right)=\int_{0}^{\infty} S(x) \cdot d x \cdot f^{M}(n(x), T(x), \varepsilon(x)),
$$

where $\mathrm{S}=\mathrm{e}^{-\mathrm{p}(\mathrm{x})}$ is an ad-hoc electron source distribution representing a more physically reasonable description than the simple point source, $S=\delta(x)$ used in [5]. This process is repeated for a range of $\varepsilon_{0}$ such that the full target $\operatorname{EDF} \mathrm{f}\left(\varepsilon_{0}\right)$ is constructed. 
This new method has a number of advantages over the simple analytic approach [5], most importantly comprising the possibility of including full descriptions of $\mathrm{T}_{\mathrm{e}}(\mathrm{x}), \mathrm{n}_{\mathrm{e}}(\mathrm{x})$ and $\phi(\mathrm{x})$ in the analysis, including the "end effects" due characterising a system of finite length and permitting the generation of a full target or sheath edge EDF. This is useful for comparison with alternative kinetic approaches and permits the construction of theoretical Langmuir probe VIcharacteristics. A number of additional refinements may also be included in applying this method, notably with regard to the validity of an argument that an electron suffering a single "last thermalising collision" is appropriate to the determination of exactly where along the parallel profile any particular electron might 'originate' (the $\mathrm{S}(\mathrm{x})$-distribution). These questions are addressed in more detail in [3].

\section{Generating theoretical Langmuir probe VI-characteristics}

For any applied voltage, $\mathrm{V}_{\text {prb}}$, the electron current to the probe is given by:

$$
\mathrm{j}_{\mathrm{e}}^{\mathrm{prb}}\left(\mathrm{V}_{\mathrm{prb}}\right)=\mathrm{n}_{0} \mathrm{e} \int_{\mathrm{w}}^{\infty} \mathrm{v}_{0} \mathrm{f}\left(\mathrm{v}_{0}\right) \mathrm{dv}_{0} / \int_{\mathrm{w}}^{\infty} \mathrm{f}\left(\mathrm{v}_{0}\right) \mathrm{dv}_{0},
$$

where $\mathrm{v}_{0}$ is the velocity at the sheath edge launching point and $\mathrm{w}=\sqrt{\frac{2}{\mathrm{~m}_{\mathrm{e}}}\left(\varepsilon_{0}^{\text {cutoff }}-\mathrm{eV}_{\mathrm{prb}}\right)}$ is the velocity corresponding to a cut-off energy, $\varepsilon_{0, \text { cutoff }}$, below which electrons cannot overcome the sheath potential for a floating probe, $\mathrm{j}_{\mathrm{i}}^{\mathrm{prb}}=\mathrm{j}_{\mathrm{e}}^{\mathrm{prb}}\left(\mathrm{V}_{\mathrm{prb}}=0\right.$, $\left.\varepsilon_{0}^{\text {cutoff }}\right)$. The ion current at the sheath edge is approximated by $\mathrm{j}_{\mathrm{i}}^{\mathrm{prb}}=\mathrm{en}_{0} \mathrm{c}_{\mathrm{s}}$ with $\mathrm{c}_{\mathrm{s}}=\sqrt{2 \mathrm{eT}_{0} / \mathrm{m}_{\mathrm{i}}}$ the sound speed.

The probe VI-characteristic can now be generated as

$$
\mathrm{j}_{\mathrm{prb}}\left(\mathrm{V}_{\mathrm{prb}}\right)=\mathrm{j}_{1}^{\mathrm{prb}}-\mathrm{j}_{\mathrm{e}}^{\mathrm{prb}}\left(\mathrm{V}_{\mathrm{prb}}\right)
$$

which can be fitted in the same way as any experimental characteristic is analysed. Examples are shown in Fig.4 for the three B2-Eirene profiles of Fig.3.

\section{Results}

Using the predicted characteristic in Fig.4 and including now additional B2-Eirene runs at intermediate values of upstream density, Fig.5 compiles the principal results of this paper. By fitting the characteristic in the voltage range pertinent to a real experimental situation $100<\mathrm{V}_{\mathrm{prb}}<0$ where here floating potential is defined as $\mathrm{V}_{\mathrm{prb}}=0$, the probe theoretical $\mathrm{T}_{\mathrm{e}}$ is derived for comparison with the values of $\mathrm{T}_{0}$ from $\mathrm{B} 2$-Eirene. Results are shown for two assumptions for the effective magnitude of $\lambda$, a critical parameter in this kinetic modelling. Open circles 
represent fits to characteristics produced from theoretical EDF's assuming the mean-free-path appropriate to "fast" test electrons in a field of background Maxwellian electrons at each $\mathrm{T}_{\mathrm{e}}(\mathrm{x})$ and $n_{e}(x)$. In this case there is very sharp break in the $n_{e}$ dependence of $T_{\text {eff }}$ for the inner target. For comparison, the filled circles correspond to the same calculations performed for $\lambda->\lambda / \lambda_{\mathrm{F}}$, where $\lambda_{\mathrm{F}}=6.5$. The factor $\lambda_{\mathrm{F}}$ originates from comparison of the simple model used here with a full PIC kinetic code [4]. These comparisons demonstrate that the value of $\lambda$ adopted in the simple model is too large. In a subsequent exercise [3], Spitzer parallel conductivity is used to estimate the parallel heat flux density, $\mathrm{q}_{\text {Spitzer }}$, for imposed $\mathrm{n}_{0}, \mathrm{~T}_{0}$ and $\mathrm{dT}_{\mathrm{e}} / \mathrm{dx}$. The result is then compared to that computed using the kinetic approach to estimate $f\left(\varepsilon_{0}\right)$. It turns out that for

$\lambda_{\mathrm{F}}=6.5, \mathrm{q}_{\text {Spitzer }}$ is within a few percent of the value predicted by the simple model. Decreasing $\lambda$ by this factor practically eliminates the rather unphysical break in the inner target $\mathrm{T}_{\text {eff }}$ of Fig.5.

Independently of the choice of $\lambda_{F}$, Fig. 5 clearly shows, that a probe at the outer target in TCV would be expected to correctly yield the local $\mathrm{T}_{\mathrm{e}}\left(\mathrm{i} . \mathrm{e} . \sim \mathrm{T}_{0}\right.$ ) at all but the lowest upstream densities. For the inner target, the choice of $\lambda_{\mathrm{F}}$ is extremely important for the predicted $\mathrm{T}_{\text {eff. }}$. For short $\lambda$, the inner target probe is predicted to read the correct local temperature throughout the density scan but would yield considerably higher values at longer values of $\lambda$.

Experimentally, TCV target probes do not appear to yield the very low values of $\mathrm{T}_{\mathrm{e}}$ indicated by B2-Eirene simulations at high density, even though the kinetic modelling applied here would suggest that they should. An alternative explanation would therefore appear to be necessary. An often cited possibility is the presence of cross-field [6] or parallel [9] resistance paths in the probe circuit. Even if the simple kinetic modelling described here does provide an adequate description of the target EDF in the presence of a strong $\mathrm{T}_{\mathrm{e}}$ gradient, the validity of the TCV B2-Eirene solutions has not been confirmed experimentally. A divertor Thomson scattering diagnostic is in preparation [12] at TCV which will hopefully permit such confirmation.

\section{Conclusions}

Simplified numerical modelling of the expected electron energy distributions at the TCV divertor target plates using simulated parallel density and temperature profiles from the B2-Eirene code shows (in Fig.5) that under conditions of high density, when the divertor plasma is in a high recycling regime or beginning to detach, target Langmuir probes should measure correctly the local target electron temperature $\mathrm{T}_{0}$. Under such conditions, $\mathrm{T}_{0}$ is predicted to be low, $1 \mathrm{eV}$, whilst the probes indicate values in excess of $5 \mathrm{eV}$ at best. At lower densities and partic- 
ularly for the TCV inner divertor target of the highly poloidally asymmetric equilibrium used as a standard diverted configuration, the target probes are expected to read considerably higher than $\mathrm{T}_{0}$ due to the contribution of weakly collisional electrons originating in regions of higher temperatures further upstream. The magnitude of this predicted $\mathrm{T}_{\mathrm{e}}$ overestimation depends strongly on the choice of mean-free-path in the simulations. Only at the lowest densities would the probe be expected again to read the local temperature, since the parallel $\mathrm{T}_{\mathrm{e}}$ profile is approximately flat. At the outer target, even at low densities, the simulated profiles of $T_{e}$ and $n_{e}$ are such that collisionality is sufficiently high for electrons from upstream, hotter regions, to be thermalised at the target and the probe should read the local temperature. The fact that this is not observed at TCV indicates that other mechanisms are responsible for this effect.

\section{Acknowledgements}

This work was partly supported by the Swiss National Science Foundation.

\section{References}

[1] R.D. Monk et al., J. Nucl. Mater. 241-243 (1997) 396-401

[2] R. A. Pitts et al., J. Nucl. Mater. 290-293 (2001) 940

[3] P. C. Stangeby, in preparation

[4] O. V. Batishchev et al., Phys. Plasmas 4(5) (1997) 1672

[5] J. A. Wesson, Plasma Phys. Contr. Fusion 37 (1995) 1459

[6] P.C. Stangeby: Plasma Phys. Contr. Fusion, 37, 1037 (1995)

[7] J.G. Watkins: J. Nucl. Mater. 266-269 (1998) 980-985

[8] R. A. Pitts, J. Horacek, A. Loarte, Bull. Am. Phys. Soc. 45 No. 7 (2000) UP1 81

[9] N. Ohno et al.: Contrib. Plasma Phys. 41 (2001) 5, 473-480

[10] D. Reiter et al., Plasma Phys. Contr. Fusion 33 (1991) 1579

[11] A.W. Leonard et al.: Phys. of Plasmas, 5, No.5, 1998

[12] R. Behn et al., Rev. Sci. Instr. 70 (1999) 768 


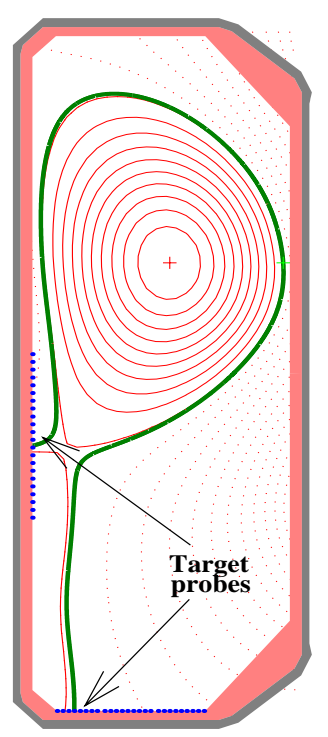

Fig.1 TCV equilibrium (\#17823, $\mathrm{I}_{\mathrm{p}}=340 \mathrm{kA}$, $\mathrm{t}=1.0 \mathrm{~s}$ ) modelled by B2-Eirene. Langmuir probe data shown in Fig.2 are obtained from this discharge. A single poloidal flux surface corresponding to a distance $1.8 \mathrm{~mm}$ from the LCFS at the outside midplane is highlighted. Parallel $\mathrm{T}_{\mathrm{e}}$ and $\mathrm{n}_{\mathrm{e}}$ profiles generated by the B2Eirene along this flux surface are used throughout this paper. 

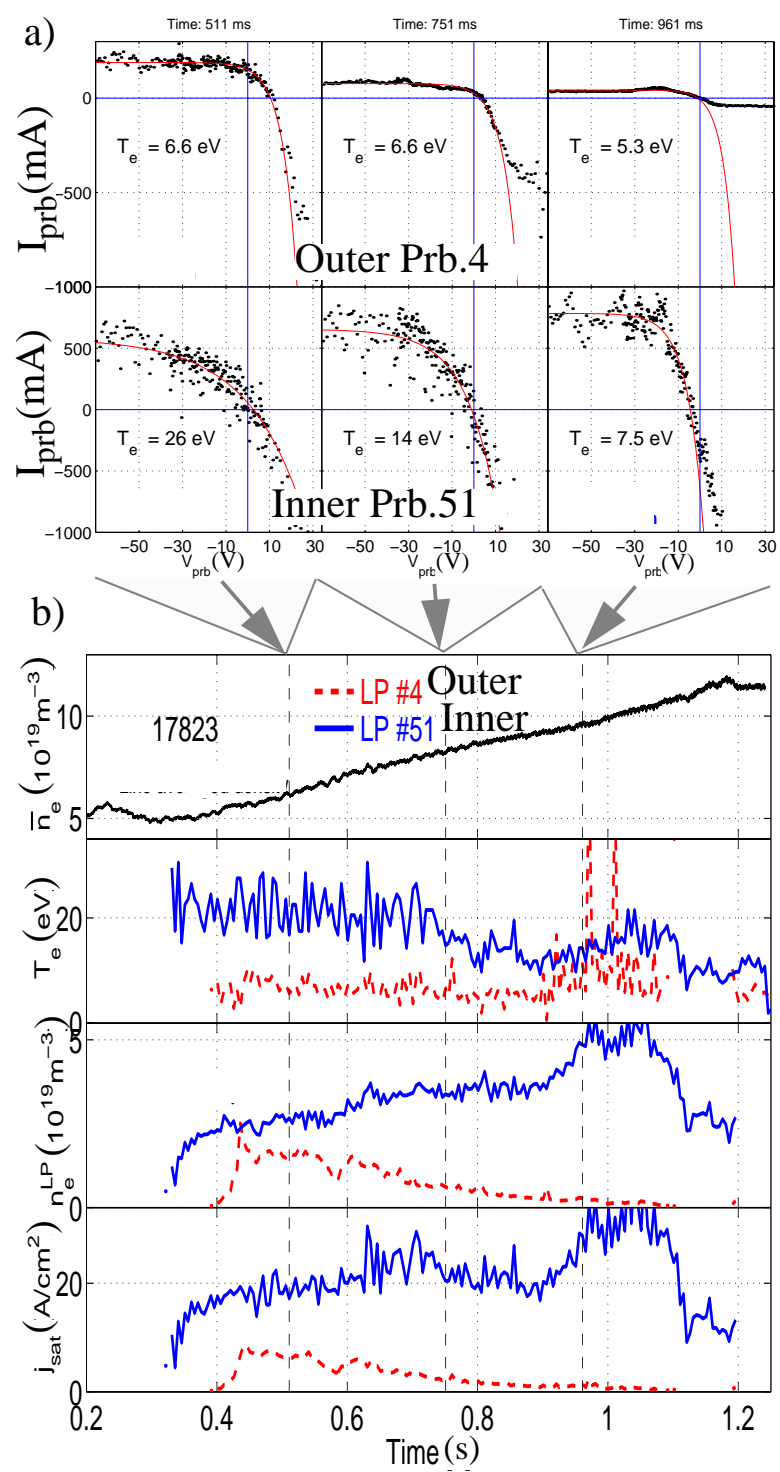

Fig.2 a) Experimental single Langmuir probe characteristics observed close the inner (\#51) and outer (\#4) target strike points for a TCV density ramp discharge. Example fitted characteristics are given at 3 different times (densities).

b) Full analysis for $T_{e}, n_{e}$, and $j_{\text {sat }}$. The vertical dashed lines indicate the times at which the characteristics in a) are plotted. 


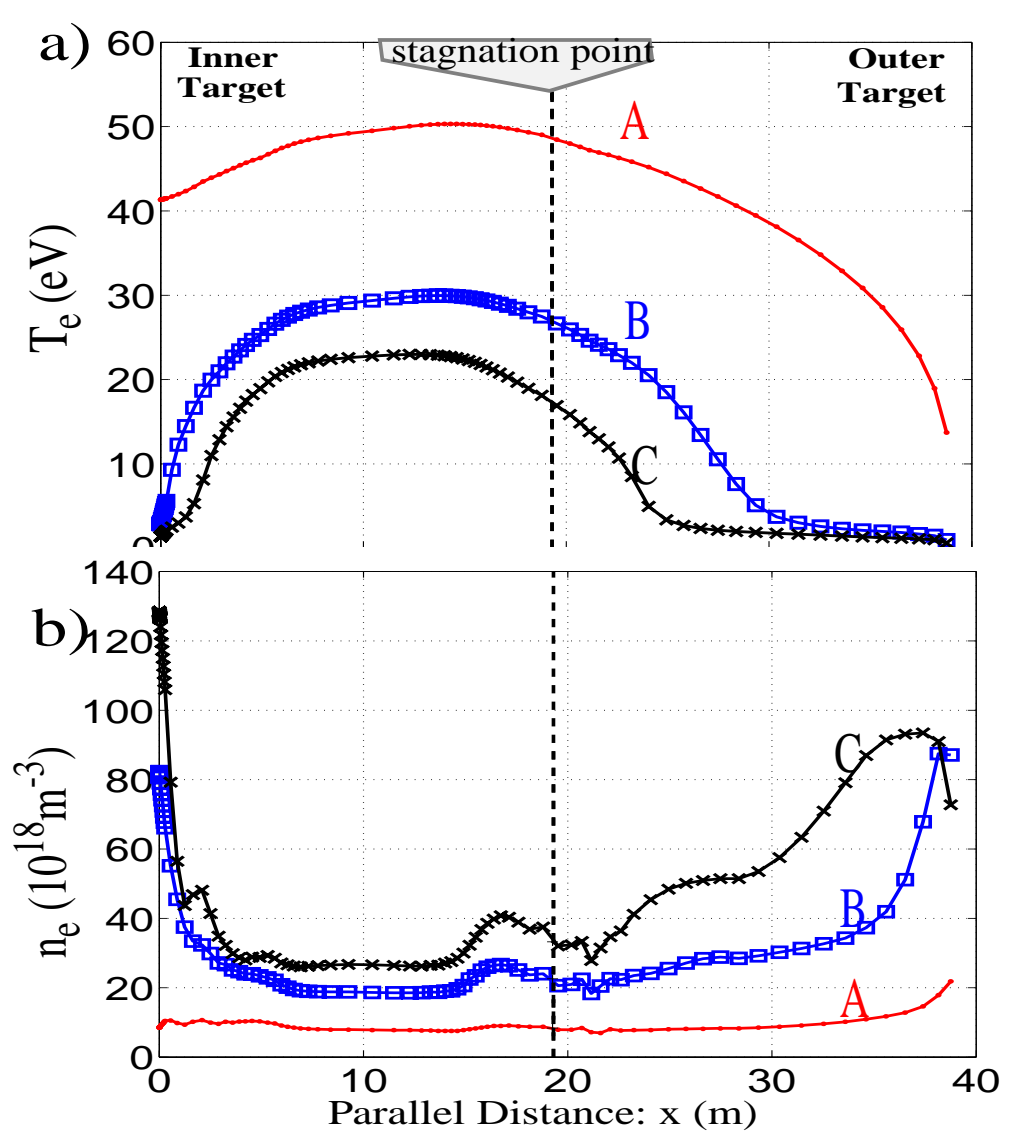

Fig.3 B2-Eirene computed parallel $\mathrm{T}_{\mathrm{e}}, \mathrm{n}_{\mathrm{e}}$ profiles for the flux surface highlighted in Fig.1. The labels A,B,C denote increasing upstream density $\mathrm{n}_{\mathrm{e}}{ }^{\mathrm{u}}=\left[8,23,33 \times 10^{18} \mathrm{~m}^{-3}\right]$, in the code. Broadly speaking the lowest density case corresponds in the code to low recycling (sheath limited) conditions, whilst the intermediate and high density are high recycling solutions. 


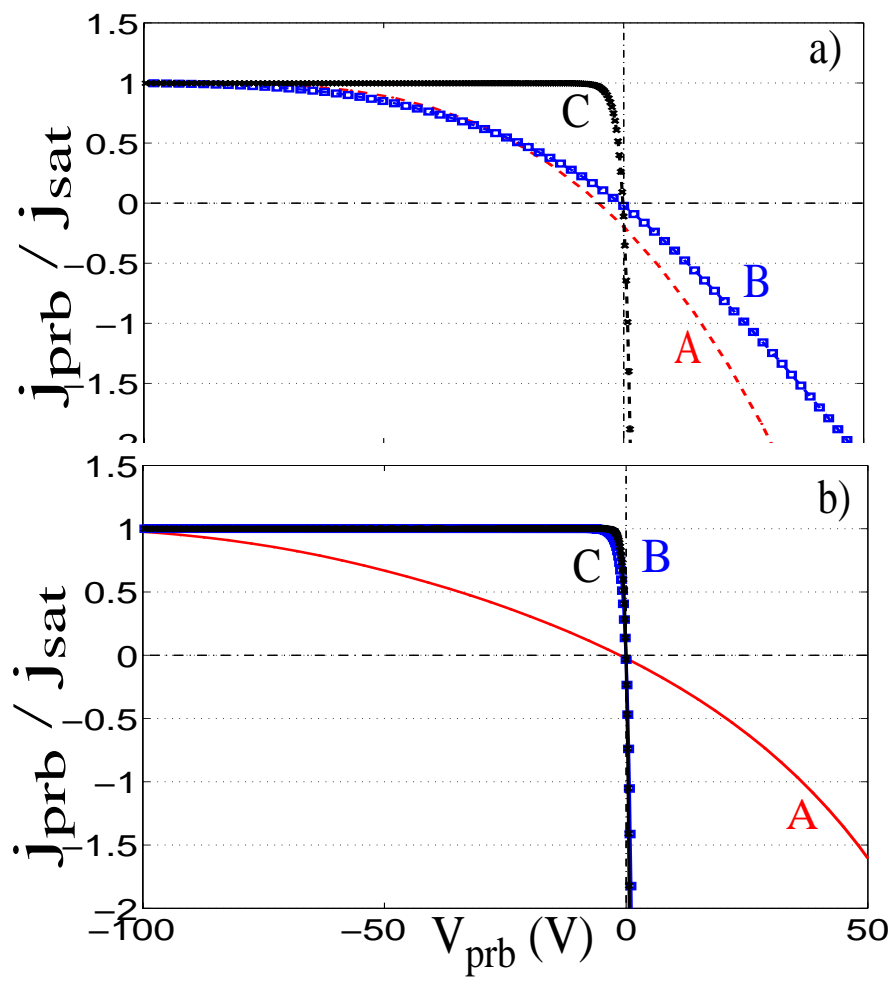

Fig.4. Calculated Langmuir probe characteristics for the inner (a) and outer (b) targets. In each case, the three sets of B2-Eirene simulated parallel field profiles of $T_{e}$ and $n_{e}$ shown in Fig.3 have been used as input for computation of the target distribution function. The probe characteristics are generated using eqn. (1) with the probe current normalised to the ion saturation current. 


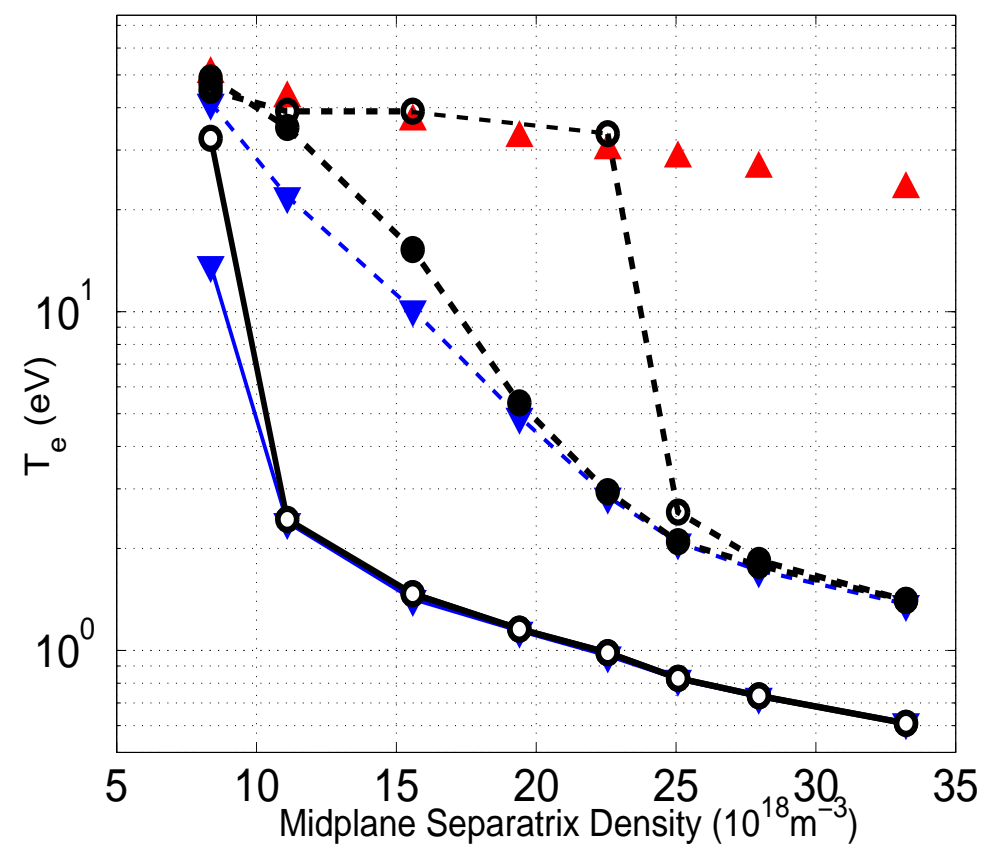

Fig.5 Density dependence of $T_{e}$ derived from an exponential fit to theoretical characteristics (Fig.4) generated using B2-Eirene simulated $\mathrm{T}_{\mathrm{e}}, \mathrm{n}_{\mathrm{e}}$ parallel profiles (Fig.3).

Full lines: outer target

Dashed lines: inner target

$\boldsymbol{\nabla}$ : B2-Eirene $\mathrm{T}_{0}$

$\boldsymbol{\Delta}$ : Max. value of $\mathrm{T}_{\mathrm{e}}$ over the B2-Eirene profile

O: $\mathrm{T}_{\mathrm{e}}^{\text {fit }}$ for $\lambda_{\mathrm{F}}=1$

O: $\mathrm{T}_{\mathrm{e}}^{\text {fit }}$ for $\lambda_{\mathrm{F}}=6.5$

The values of $T_{0}$ and $T_{\max }$ represent lower and upper limit for the $\mathrm{T}_{\mathrm{e}}^{\text {fit }}$. Note $\mathrm{T}_{\mathrm{eff}}=\mathrm{T}_{\mathrm{e}}{ }^{\text {fit }} / \mathrm{T}_{0}$. 